Case Report

\title{
Patient Factors Influencing Adherence to ART Treatment among HIVIAIDS Patients in Embu Teaching and Referral Hospital Comprehensive Care Clinic
}

\author{
Evangeline Kananu Njue Mugoh ${ }^{1, *}$, Ephantus Kabiru ${ }^{1}$, Joyce Mwaniki \\ ${ }^{1}$ Department of Community Health, Kenyatta University, Nairobi, Kenya \\ ${ }^{2}$ Department of Plant Sciences, Kenyatta University, Nairobi, Kenya \\ Email address: \\ evangelinemugoh@gmail.com (E. K. N. Mugoh), ewkabiru@yahoo.com (E. Kabiru), joycemwaniki@gmail.com (J. Mwaniki) \\ ${ }^{*}$ Corresponding author
}

To cite this article:

Evangeline Kananu Njue Mugoh, Ephantus Kabiru, Joyce Mwaniki. Patient Factors Influencing Adherence to ART Treatment among HIV/AIDS Patients in Embu Teaching and Referral Hospital Comprehensive Care Clinic. Science Journal of Public Health.

Vol. 4, No. 5, 2016, pp. 375-380. doi: 10.11648/j.sjph.20160405.12

Received: July 18, 2016; Accepted: July 28, 2016; Published: August 17, 2016

\begin{abstract}
Adherence to antiretroviral therapy is a major predictor of the survival of individuals living with HIV/AIDS. Appropriate use of antiretroviral drugs (ARVs) has improved the health of many HIV positive individuals. The effectiveness of HIV treatment depends on sustenance of high levels of adherence to ARVs, however, ARV regimens are often complicated and can be affected by varying dosing schedules, failing to have proper dietary requirements and patients developing adverse effects. The main objective of this study was to determine patient's demographic, social and economic factors influencing adherence to Anti-retroviral drugs among HIV/AIDS adult patients. A cross sectional study was carried out at Embu County Teaching and Referral Hospital in Kenya. Stratified random sampling was used to obtain relative proportion of male and female respondents. 332 HIV positive patients were chosen from a total of 1694 patients who were active in ART for more than one year. A semi-structured interview schedules was used to obtain information. The study revealed that almost half (48.2\%) of the respondents had optimal adherence to ART treatment. $34.9 \%$ cited traveling as an hindrance to optimal adherence to treatment. Adherence to ART treatment was significantly associated with age of the respondents, marital status, main occupation and average monthly income of the respondents. The study recommends that HIV patients should be given more information on the importance of consistency and nearly perfect adherence to ART. The patients should be enlightened on the importance of carrying their ARV drugs even when going for short travels.
\end{abstract}

Keywords: ART Adherence, Demographic Characteristics, Economic Characteristics, Social Factors

\section{Introduction}

\subsection{Background Information}

Adherence to ART is crucial for treatment success among HIV patients, high levels of adherence is a prerequisite for maintained viral suppression and lowers risk of drug resistance, this in turn will prevent premature morbidity and mortality [1]. Low adherence is the second strongest determinant for disease deterioration and death after CD4 count. Non-adherence to ART is a substantial challenge in resource-poor settings where increasing drug resistance is hard to combat using the limited treatment alternatives available [2]. It is estimated that the average rates of non-adherence to antiretroviral therapy to be ranging between $50 \%$ and $70 \%[3]$.

Globally the number of people living with HIV/AIDS continues to grow, for instance, there were 36.9 million people living with HIV in 2014, up from 29.8 million in 2001, this is as a result of continued new infections, people living longer with HIV and general population growth. The number of patients on antiretroviral therapy worldwide increased from 
7.5 million in 2010 to over 15 million in 2015 representing $72 \%$ of eligible patients according to WHO 2013 guidelines.

\subsection{Problem Statement}

Significant progress has been made since lifesaving drugs for HIV/AIDS came into existence. Low compliance to prescribed medical interventions is an ever present and complex problem, especially for patients with a chronic illness. With an increasing number of medications shown to do more good than harm when taken as prescribed, low compliance is a growing concern and seriously undermines the benefits of the current medical care.

Embu County, as of 2014, had an HIV prevalence of 3.7\% with $80.8 \%$ (3718 out of 4600) of adult PLHIV eligible for ART having access to treatment [6]. There has been no documented study on the adherence levels among HIV patients living with HIV/AIDS or on the factors influencing their adherence. Therefore this study sought to explore patient factors that influence adherence to ART among AIDS patients in Embu.

\subsection{Objectives}

i. To determine the proportion of HIV/AIDS patients that adhered to ART treatment among HIV patients attending Embu County Teaching and Referral Hospital Comprehensive Care Clinic.

ii. To establish socio-demographic and economic factors that influence adherence to ART drugs among HIV patients attending Embu County Teaching and Referral Hospital Comprehensive Care Clinic.

\section{Literature Review}

\subsection{HIV and AIDS Management in Kenya}

More than 1.5 million people were infected with HIV in Kenya by the year 2005 but in the year 2012, 1.6 million people were living with HIV with a prevalence rate of $6.1 \%$ [7, 8] Kenyan new HIV infections are estimated to have stabilized at an average of 89,000 among adults and about 11,000 among children annually. However progress has been made with HIV prevalence dropping from $10.5 \%$ in 1996 to $6.1 \%$ in 2012 but HIV, continues to contribute the highest mortality rates, increase households burden and straining national health systems [8]. In the Kenya Aids Strategic Framework $2014 / 2015$ - 2018/2019, Kenya aims at reducing new HIV infections by $75 \%$ and reduce AIDS related mortality by $25 \%$ in the next five years [8]. In addition, Kenya's Vision 2030 aims at achieving comprehensive HIV prevention, treatment and care.

Of the 1.6 million PLHIV, 656,359 were on ART against estimated 902,302 in 2014 (141,608 children and 760,694 adults) PLHIV needing ART according to WHO 2009 guidelines [10] while according to the Kenya AIDs Indicator Survey $2014,78.4 \%$ of adults and $42.5 \%$ of children eligible for ART coverage were receiving treatment as of year 2013 [11]. The low uptake in children was because of poor awareness on the part of parents and caregivers than non-availability of drugs.

\subsection{Adherence of ART Treatment Among HIV/AIDS Patients}

Maximum and sustainable suppression of HIV viral replication to below the level of detection is necessary to achieve these biological and clinical goals. For success to be achieved a near-perfect adherence to combination ARV regimens is very necessary. Consistency and nearly perfect adherence is considered an essential requirement for HIV positive patients on ART to fully realize its life extending benefits [2, 12]. Any levels of adherence below 95\% have been associated with poor suppression of HIV viral load and a lowering of CD4 count leading to disease progression and development of drug resistance [13]. Evidence suggests that adherence greater than $95 \%$ is necessary to adequately suppress viral replication and produce a durable response and stop disease progression.

\section{Methodology}

This was a descriptive cross sectional research that utilized quantitative research method of data collection and analysis conducted in Embu County Hospital comprehensive care clinic among HIV adult that were active in ART for more than one year. The sample size of 332 patients utilized. Stratified sampling was used to sample patients. Data was collected using face-to-face interviews using structured questionnaires. Quality control measures were employed including pre-test, validity and reliability checks.

Authority to carry out the study was sought from NACOSTI. Approval to conduct research was sought from Kenyatta University Ethical Review Committee; and clearance from Embu Teaching and Referral Hospital and Embu County authorities. Respondents consented to participate with confirmation of confidentiality. Collected data was cross-checked for completeness and any missing entries corrected upon completion of each interview.

Data coding, entry and analysis was done using the SPSS version 20 software. The questionnaires were inspected to detect errors and omissions and corrected immediately. Multiple binary logistic regression used to test the association between ART adherence and demographic and socio-economic factors. Findings were presented as text, graphs and tables.

\section{Findings and Discussion}

\subsection{Adherence to ART Treatment}

Both methods of evaluating adherence indicated that 160 patients had optimal adherence to treatment. However there were 11 patients which according to pill count had sub-optimal adherence to treatment while they evaluated themselves as having $100 \%$ adherence. And therefore because the pill-counts is considered the most 'objective' of the approach to assessing ART adherence, the 160 (48.2\%) were considered to have had optimal ART adherence and the rest having sub-optimal 
adherence to ART as shown in Table 1. This was different from a study by Kidder et al [15] where in a much higher figure $84.6 \%$ of 30 -days optimal adherence was reported in a longitudinal group-randomized trial in Kenya. A study done in Pumwani Hospital by Karanja [16] reported higher levels of adherence, where $91 \%$ of the respondents showed perfect adherence levels between 95 and 100\% according to hospital records as opposed to this study in Embu comprehensive care clinic. Similar findings were made among HIV patients attending Moi Teaching and Referral Hospital where it was reported that the overall adherence levels based on timing of taking ARVs was low for all respondents $43.2 \%$, clearly indicated that there were serious barriers to adherence [17].

Table 1. Comparison between Self-report and Pill Count.

\begin{tabular}{lll}
\hline $\begin{array}{l}\text { Self-report adherence in } \\
\text { the last one month }\end{array}$ & \multicolumn{2}{c}{ Adherence to ART treatment (pill count) } \\
\cline { 2 - 3 } & Sub-optimal & Optimal \\
\hline $10 \mathrm{~cm}$ & 11 & 160 \\
$9 \mathrm{~cm}$ & 118 & 0 \\
$8 \mathrm{~cm}$ & 31 & 0 \\
$<=7 \mathrm{~cm}$ & 12 & 0 \\
\hline
\end{tabular}

\subsection{Causes of Non-adherence}

According to figure 1 below, 116 (34.9\%) of the respondents travelling was considered as the main cause of hindrance to optimal adherence to treatment. Other reasons included forgetfulness (23.5\%), fatigue of taking drugs all the time $(20.8 \%)$ and side effects $20.8 \%$. Similar findings were made in Southwest Ethiopia where most (43.7\%) simply indicated forgetfulness as the main hindrance to ART adherence. Similar findings were made in Pumwani CCCs where it was found out that most patients cited forgetfulness and side effects as a challenge in taking medications [16]. Similar findings were also made at Moi Teaching and Referral Hospital where it was found out that patients failed to adhere to timing of drugs because of being away from home (58.7\%), forgetting $(59.0 \%)$, change in routine $(59.6 \%)$, and running out of medicines $(60.9 \%)$ and due to unpleasant taste of ARVs
(65.2\%). A much lower proportions were reported in Botswana where $58 \%$ of ARV users who reported having experienced side-effects, only $8 \%$ cited side-effects as a reason for failure to achieve optimal adherence [13].

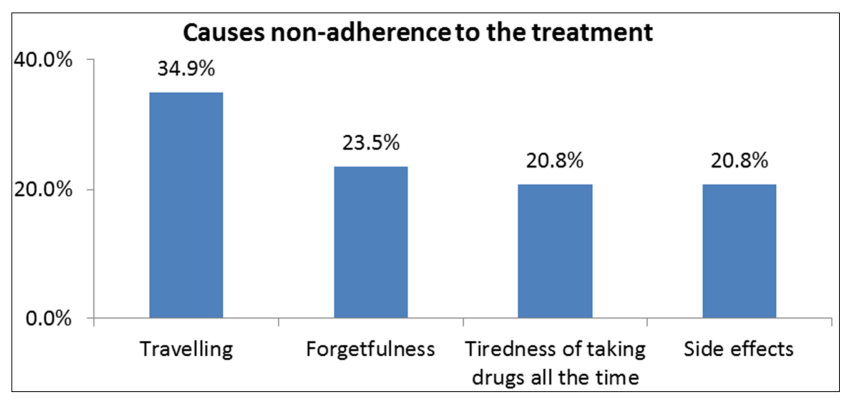

Figure 1. Causes non-adherence to the treatment.

\subsection{Influence of Demographic Factors on ART Adherence}

As shown in Table 2, adherence to ART treatment was significantly associated with age of the respondents with respondents aged below 25 years 4.028 times more likely (Adjusted Odds Ratio $=4.028, \mathrm{p}$-value $=0.048$ ) to have optimal ART adherence as compared to respondents aged 56 years and above. Marital status was also significantly associated with ART adherence with monogamously married and polygamous married respondents more likely to have optimal adherence as compared to widowed or widower respondents. Contrary findings were made in a cohort study in Kenya by Karcher et al. [19] Where older patients were found to have lower treatment denial and therefore a higher risk of mortality as compared to younger patients. Similar findings were also made in Moi Teaching and Referral Hospital in which it was found out that most of the patients who failed to adhere to ART treatment were in the age bracket of 36-45 years and were either divorced, widowed or separated [17]. Contrary findings were made in Ethiopia where it was founnd that married patients had least adherence to ART treatment [20].

Table 2. Influence of Socio-demographic factors on ART adherence.

\begin{tabular}{|c|c|c|c|c|c|}
\hline \multirow{2}{*}{ Demographic characteristic } & \multirow{2}{*}{ Categories } & \multicolumn{2}{|c|}{ Adherence to ART treatment } & \multirow{2}{*}{$\operatorname{AOR}(95 \% \mathrm{CI})$} & \multirow{2}{*}{ P-value } \\
\hline & & Sub-optimal & Optimal & & \\
\hline \multirow{5}{*}{ Age of respondent } & $18-25$ & $5(23.8)$ & $16(76.2)$ & $4.028(1.011-16.050)$ & .048 \\
\hline & $26-35$ & $34(46.6)$ & $39(53.4)$ & $1.233(.465-3.267)$ & .674 \\
\hline & $36-45$ & $60(51.7)$ & $56(48.3)$ & $1.061(.425-2.647)$ & .899 \\
\hline & $46-55$ & $52(61.2)$ & $33(38.8)$ & $.824(.336-2.022)$ & .672 \\
\hline & 56 and above (Ref) & $21(56.8)$ & $16(43.2)$ & & \\
\hline \multirow{2}{*}{ Gender of respondent } & Male & $50(47.6)$ & $55(52.4)$ & $1.013(.607-1.690)$ & .962 \\
\hline & Female (Ref) & $122(53.7)$ & $105(46.3)$ & & \\
\hline \multirow{5}{*}{ Education level of respondents } & Primary & $50(50.5)$ & $49(49.5)$ & $.858(.295-2.496)$ & .779 \\
\hline & Secondary & $72(49.0)$ & $75(51.0)$ & $.690(.228-2.086)$ & .511 \\
\hline & Mid-level College & $29(58.0)$ & $21(42.0)$ & $.516(.156-1.707)$ & .278 \\
\hline & University & $7(58.3)$ & $5(41.7)$ & $.507(.103-2.498)$ & .404 \\
\hline & None (Ref) & $14(58.3)$ & $10(41.7)$ & & \\
\hline \multirow{5}{*}{ Marital status of respondents } & Single & $48(52.7)$ & $43(47.3)$ & $2.085(.873-4.984)$ & .098 \\
\hline & Married Monogamous & $56(44.4)$ & $70(55.6)$ & $3.744(1.665-8.420)$ & .001 \\
\hline & Married polygamous & $16(38.1)$ & $26(61.9)$ & $4.235(1.645-10.903)$ & .003 \\
\hline & Separated/Divorced & $11(61.1)$ & $7(38.9)$ & $1.890(.601-5.943)$ & .276 \\
\hline & Widow/er (Ref) & $41(74.5)$ & $14(25.5)$ & & \\
\hline
\end{tabular}




\subsection{Influence of Social Factors on ART Adherence}

The respondents who had disclosed their HIV status was significantly associated with ART adherence, respondents who had disclosed their HIV status to spouse or sexual partner were 3.525 times more likely (Adjusted Odds Ratio $=3.525$, $\mathrm{p}$-value $=0.008)$ and those who had disclosed to parents or children were more likely (Adjusted Odds Ratio $=2.779$, p-value $=0.05)$ to adhere to ART treatment as compared to those who had disclosed their HIV status to friends or colleagues as indicated in Table 3. Non-disclosure to family members and friends is seen as a constraint to successful adherence and in cases of non-disclosure among partners, the partner on ART may resort to hiding pills, occasionally skipping medications and failure to keep clinic appointments for refills or review while disclosure on the other hand allow for support which plays a vital role in encouraging good adherence [21]. In Southern Malawi, although healthcare providers advocate for disclosure of HIV status to family members, more than half of the providers interviewed stated that some of the women fear disclosing their HIV status to their spouses because their husbands may leave them [22]. A study at Pumwani CCCs reported that among patients interviewed all had disclosed their HIV status to someone close to them while most reported to be receiving support from the people they disclosed to [16].

In this study presence of psychological or emotional support was not associated with ART adherence. Similar findings were made in Ethiopia where it was reported that presence of support from family did not translate to better ART adherence as patients might miss support at working places and other places outside home [20]. Contrary findings were made in Malawi where it was also revealed that there was better adherence among patients who get some form of support in their homes, hospital, or among their friends, as compared with non-supported patients [22]. Contrary findings were also made in South-west Ethiopia where it was found that existence of social support was a significant predictor of adherence to treatment.

Table 3. Influence of Social Factors on ART Adherence.

\begin{tabular}{|c|c|c|c|c|c|}
\hline \multirow{2}{*}{ Social factors } & \multirow{2}{*}{ Categories } & \multicolumn{2}{|c|}{ Adherence to ART treatment } & \multirow{2}{*}{$\operatorname{AOR}(95 \% \mathrm{CI})$} & \multirow{2}{*}{ P-value } \\
\hline & & Sub-optimal & Optimal & & \\
\hline \multirow{2}{*}{ Disclosed HIV status } & Yes & $149(51.0)$ & $143(49.0)$ & $1.298(.666-2.532)$ & .443 \\
\hline & No (Ref) & $23(57.5)$ & $17(42.5)$ & & \\
\hline \multirow{4}{*}{ Relationship with person disclosed to HIV status } & Spouse/Sexual partner & $56(40.9)$ & $81(59.1)$ & $3.525(1.395-8.909)$ & .008 \\
\hline & Parent/Children & $26(44.8)$ & $32(55.2)$ & $2.779(.999-7.733)$ & .050 \\
\hline & Other Relatives & $42(65.6)$ & $22(34.4)$ & $1.278(.464-3.519)$ & .635 \\
\hline & Friends/Colleagues (Ref) & $25(75.8)$ & $8(24.2)$ & & \\
\hline \multirow{3}{*}{ Type of support received } & Financial & $26(47.3)$ & $29(52.7)$ & $1.479(.419-5.219)$ & .543 \\
\hline & Moral & $68(46.9)$ & $77(53.1)$ & $2.102(.643-6.873)$ & .219 \\
\hline & Spiritual (Ref) & $13(72.2)$ & $5(27.8)$ & & \\
\hline \multirow{3}{*}{ Rate support received } & Excellent & $72(41.6)$ & $101(58.4)$ & $.968(.396-2.368)$ & .943 \\
\hline & Good & $64(69.6)$ & $28(30.4)$ & $.342(.132-.886)$ & .127 \\
\hline & Poor (Ref) & $13(48.1)$ & $14(51.9)$ & & \\
\hline ART opinion & Approve & $168(51.5)$ & $158(48.5)$ & $2.729(.402-18.522)$ & .304 \\
\hline Friends or relatives avoidance & No (Ref) & $124(50.8)$ & $120(49.2)$ & & \\
\hline \multirow{2}{*}{ Family or community support in taking ARV } & Yes & $135(51.9)$ & $125(48.1)$ & $.863(.443-1.683)$ & .666 \\
\hline & No (Ref) & $37(51.4)$ & $35(48.6)$ & & \\
\hline
\end{tabular}

\subsection{Influence of Economic Factors on ART Adherence}

Main occupation of the respondents was significantly associated with ART adherence with employed 0.356 times less likely (Adjusted Odds Ratio $=0.356$, $\mathrm{p}$-value $=0.016$ ), casual workers 0.264 times less likely (Adjusted Odds Ratio $=0.264$, $\mathrm{p}$-value $=0.04)$ and those engaged in business 0.328 times less likely (Adjusted Odds Ratio $=0.328, \mathrm{p}$-value $=$ 0.016) to adhere to ART treatment as compared to students or those who were unemployed as indicated in Table 4. Similar findings were made in Ethiopia where it was observed that unemployed patients were more likely to be depressed and less likely to socialize, leading to miss appointments and access to health care [23]. Similar findings were also made in Cameron where it was reported that adherence was high among employed patients as compared to unemployed patients [24]. Similar findings has been observed in low-income countries where it was found that overall pooled odds ratio (OR) for the association between being employed and adhering to ART was 1.85 [25].

Level of income was also significantly associated with ART treatment adherence with respondents earning less than Kshs 5,000 and those earning between Kshs 5,000 and Kshs 10,000 more likely to adhere to treatment as compared to respondents earning more Kshs 15,000 . This showed that patients earning less had better adherence as compared to higher income earners. Contrary findings were made in Cameron where it was observed that a monthly middle income was significantly associated with greater pharmacy adherence. Contrary findings were also made in Pumwani CCCs where respondents earning an income of 20,000 Kenyan shillings and above were four times more likely to adhere to ART which 
was attributed to the ability to meet expenses such as transport with economic problems that can negatively affect adherence. to the clinic, food and shelter leading to a better way of dealing

Table 4. Influence of Economic Factors on ART Adherence.

\begin{tabular}{|c|c|c|c|c|c|}
\hline \multirow{2}{*}{ Economic factors } & \multirow{2}{*}{ Categories } & \multicolumn{2}{|c|}{ Adherence to ART treatment } & \multirow{2}{*}{$\operatorname{AOR}(95 \% \mathrm{CI})$} & \multirow{2}{*}{ P-value } \\
\hline & & Sub-optimal & Optimal & & \\
\hline \multirow{5}{*}{ Main occupation } & Employed & $55(52.4)$ & $50(47.6)$ & $.356(.154-.822)$ & .016 \\
\hline & Casual worker & $34(61.8)$ & $21(38.2)$ & $.264(.108-.648)$ & .004 \\
\hline & Farmer & $33(52.4)$ & $30(47.6)$ & $.486(.202-1.171)$ & .108 \\
\hline & Business & $37(53.6)$ & $32(46.4)$ & $.328(.135-.800)$ & .014 \\
\hline & Student/unemployed (Ref) & $13(32.5)$ & $27(67.5)$ & & \\
\hline \multirow{4}{*}{ Monthly income (Kshs.) } & $\leq 5,000$ & $76(55.1)$ & $62(44.9)$ & $2.937(1.032-8.359)$ & .044 \\
\hline & $5,001-10,000$ & $47(46.1)$ & $55(53.9)$ & $4.213(1.453-12.215)$ & .008 \\
\hline & $10,001-15,000$ & $18(62.1)$ & $11(37.9)$ & $2.200(.635-7.623)$ & .214 \\
\hline & $>15,000$ (Ref) & $18(78.3)$ & $5(21.7)$ & & \\
\hline \multirow{3}{*}{ Main source of food } & Market \& grocery (Ref) & $107(49.8)$ & $108(50.2)$ & & \\
\hline & Own farm & $62(56.4)$ & $48(43.6)$ & $.612(.349-1.074)$ & .087 \\
\hline & Others & $3(42.9)$ & $4(57.1)$ & $.622(.121-3.184)$ & .568 \\
\hline \multirow{3}{*}{ Number of meals per day } & One meal & $4(57.1)$ & $3(42.9)$ & $.710(.151-3.333)$ & .664 \\
\hline & Two meals & $40(51.9)$ & $37(48.1)$ & $.912(.527-1.580)$ & .743 \\
\hline & Three meals (Ref) & $128(51.6)$ & $120(48.4)$ & & \\
\hline
\end{tabular}

\section{Conclusions}

Based on the study findings the researcher concludes as follows:

1. About half of the patients had optimal adherence to ART treatment while slightly over half of the patients had sub-optimal adherence to ART treatment.

2. Age, marital status, main source of income and average monthly influences adherence to treatment and majority had disclosed their HIV to significantly others of whom they had received satisfactory moral support. Disclosure of HIV status to the spouse or sexual partner increases ART adherence.

\section{Recommendations}

Based on the study findings the researcher recommends as follows:

1. HIV patients should be given more information on the importance of consistency and nearly perfect adherence to ART so as to fully realize its life extending benefits as it has been shown that there is significant non-adherence among HIV positive patients on ART in the County.

2. HIV patients should be enlightened on the importance of carrying their ARV drugs even when going for short travels so that they will not miss their doses. Additionally the study recommends should be enlightened on having treatment assistants/buddies who can help them remember to take the treatments all the time.

\section{Acknowledgments}

The authors of this publication "Patient Factors Influencing Adherence to ART Treatment among HIV/AIDS Patients in Embu Teaching and Referral Hospital Comprehensive Care Clinic" would like to thank Kenyatta University for supervision; Embu County Teaching and Referral Hospital for permission and Exactitude Research Consultants (Ltd) for data management services.

\section{Conflict of Interest}

All authors report no conflicts of interest in this publication.

\section{Authors' Contributions}

Authors made substantial contributions to conception and design, and/or acquisition of data, and/or analysis and interpretation of data.

\section{References}

[1] Christian Unge et al., "Long-Term Adherence to Antiretroviral Treatment and Program Drop-Out in a High-Risk Urban Setting in Sub-Saharan Africa: A Prospective Cohort Study," PLOS ONE, vol. 5, no. 10, p. e 13613, 2010, doi: 10.1371/journal.pone.0013613.

[2] Michael L Scanlon and Rachel C Vreeman, "Current strategies for improving access and adherence to antiretroviral therapies in resource-limited settings," HIV AIDS, vol. 5, pp. 1-17, 2013.

[3] World Health Organization [WHO], "From Access to Adherence: The Challenges of Antiretroviral Treatment Studies from Botswana, Tanzania and Uganda," World Health Organization, Geneva, 2006.

[4] Kaiser Family Foundation. (2015, July) Kaiser Family Foundation. [Online]. http://kff.org/global-health-policy/fact-sheet/the-global-hivaid s-epidemic/\#footnote-UNAIDSGlobalReport

[5] UNAIDs. (2015) UNAIDs. [Online]. http://www.unaids.org/sites/default/files/media_asset/MDG6R eport_en.pdf

[6] NASCOP \& NACC, "Kenya HIV County Profiles," Nairobi, 2014. 
[7] UNICEF. (2013, December) UNICEF Kenya. [Online]. http://www.unicef.org/infobycountry/kenya_statistics.html

[8] Avert. (2015) Avert. [Online]. http://www.avert.org/professionals/hiv-around-world/sub-saha ran-africa/kenya

[9] NACC, "Kenya AIDS Strategic Framework 2014/2015 2018/2019," National AIDS Control Council, Ministry of Health, 2014.

[10] NACC, "Kenya AIDS Response Progress Report 2014: Progress towards Zero," Nairobi, 2014.

[11] NASCOP, "Kenya HIV Estimates: June 2014," Nairobi, 2014.

[12] C. Shumba, L. Atuhaire, R. Imakit, R. Atukunda, and P. Memiah, "Missed Doses and Missed Appointments: Adherence to ART among Adult Patients in Uganda," International Scholarly Research Notices, vol. 2013, no. 2013, 2013.

[13] Joyce Kgatlwane et al., "Factors that facilitate or constrain adherence to antiretroviral therapy among adults at four public health facilities in Botswana: a pre-intervention study," 2006.

[14] Mathieu Rougemont, Beat E Stoll, Nadia Elia, and Peter Ngang, "Antiretroviral treatment adherence and its determinants in Sub-Saharan Africa: a prospective study at Yaounde Central Hospital, Cameroon," AIDS Research and Therapy, vol. 6, no. 21, October 2009.

[15] Daniel P. Kidder et al., "HIV Prevention in Care and Treatment Settings: Baseline Risk Behaviors among HIV Patients in Kenya, Namibia, and Tanzania," PLoS ONE, vol. 8, no. 2, p. e 57215, February 2013.

[16] Sarah Wangui Karanja, "Factors Influencing Adherence to Antiretroviral Medications Among Patients Living With HIV in Kenya," Department of Sociology and Social Work, University of Nairobi, Thesis 2013.

[17] N. C Talam, P. Gatongi, J. Rotich, and S. Kimaiyo, "Factors Affecting Antiretroviral Drug Adherence Among HIV/AIDS Adult Patients Attending HIV/AIDS Clinic at Moi Teaching and Referral Hospital, Eldoret, Kenya.," East African Journal of Public Health, vol. 5, no. 2, pp. 74-78, August 2008.
[18] Alemayehu Amberbir, Kifle Woldemichael, Sofonias Getachew, Belaineh Girma, and Kebede Deribe, "Predictors of adherence to antiretroviral therapy among HIV-infected persons: a prospective study in Southwest Ethiopia," BMC Public Health, vol. 8, no. 265, July 2008.

[19] Heiko Karcher, Austin Omondi, John Odera, Andrea Kunz, and Gundel Harms, "Risk factors for treatment denial and loss to follow-up in an antiretroviral treatment cohort in Kenya," Tropical Medicine \& International Health, vol. 12, no. 5, pp. 687-694, May 2007.

[20] Habtamu Mitiku, Tekabe Abdosh, and Zelalem Teklemariam, "Factors Affecting Adherence to Antiretroviral Treatment in Harari National Regional State, Eastern Ethiopia," ISRN AIDS, vol. 960954, 2013, doi: 10.1155/2013/960954.

[21] Bentsi Sam Ophelia, "Factors That Constrain Adherence to Antiretroviral Therapy among HIV Positive Patients in the Sekondi-Takoradi Metropolis," School of Public Health, Kwame Nkrumah University of Science and Technology, Master's Thesis 2015.

[22] Ogbochi McKinney, Naomi N. Modeste, Jerry W. Lee, Peter C. Gleason, and Gisele Maynard-Tucker, "Determinants of Antiretroviral Therapy Adherence among Women in Southern Malawi: Healthcare Providers' Perspectives," AIDS Research and Treatment, vol. 2014, no. 2014, December 2014.

[23] Bayew Tsega, Bhagavathula Akshaya Srikanth, and Zewdneh Shewamene, "Determinants of non-adherence to antiretroviral therapy in adult hospitalized patients, Northwest Ethiopia," Patient Prefer Adherence, vol. 9, pp. 373-380, 2015, doi: 10.2147/PPA.S 75876 .

[24] Patou Masika Musumari et al., "Food Insecurity is Associated with Increased Risk of Non-Adherence to Antiretroviral Therapy among HIV-Infected Adults in the Democratic Republic of Congo: A Cross-Sectional Study," PLoS ONE, vol. 9, no. 1, p. e 85327, 2014, doi: 10.1371/journal.pone.0085327.

[25] Jean B Nachega et al., "Association between antiretroviral therapy adherence and employment status: systematic review and meta-analysis," Bulletin of the World Health Organization, vol. 93, no. 1, pp. 29-41, 2014, doi: 10.2471/BLT.14.138149. 